\title{
Agenesia de la arteria pulmonar izquierda: reporte de caso
}

\section{Agenesis of the left pulmonary artery: a case report}

\author{
Juan David Cáceres González ${ }^{1}$, Manuel Felipe Cáceres-Acosta² ${ }^{2}$ Jovani Osorno Serna ${ }^{3}$, Gustavo
} Adolfo Gómez Correa ${ }^{4}$, Fredy Alexander Rodríguez Reyes ${ }^{5}$, Tatiana Suarez-Poveda ${ }^{6}$

\section{Resumen}

Se orienta en este reporte de caso sobre la importancia que tiene la sospecha diagnóstica de un cuadro patológico inusual y que con una rápida detección puede generar beneficios considerables en el paciente que lo padece. Se presenta el caso de una mujer de 21 años con antecedentes patológicos de asma en la niñez, quién cursó hace seis meses con cuadro neumónico. La paciente consultó por dorsalgia continua hace dos meses, asociado a cuatro episodios de hemoptisis. En la Tomografía Axial Computarizada (TAC) contrastada de tórax se evidenció ausencia de la arteria pulmonar izquierda. En conclusión, la agenesia unilateral de la arteria pulmonar se convierte en un reto diagnóstico por su poca prevalencia y sus diversas manifestaciones clínicas, siendo relevante su identificación temprana.

Palabras clave: agenesia; arteria pulmonar; hemoptisis.

\begin{abstract}
It is intended to guide through a case report the importance of the diagnostic suspicion of an unusual pathological condition, but with a fast detection it can generate considerable benefits to the patient who suffers it. A 21-year-old female with a history of childhood asthma and who presented a pneumonic episode of 6 months of evolution. The patient consulted for continuous back pain of 2 months associated with 4 episodes of hemoptysis. A chest computed tomography scan (CT scan) revealed absence of the left pulmonary artery. In conclusion, the unilateral agenesis of the pulmonary artery becomes a diagnostic challenge due to its low prevalence and diverse clinical manifestations, being relevant an early identification.
\end{abstract}

Keywords: agenesis, pulmonary artery, hemoptysis.
${ }^{1}$ Residente de Medicina Interna Universidad del Valle. Cali, Colombia.

${ }^{2}$ Especialista en Medicina Interna y Cardiología. Docente del Departamento de Medicina Interna y Cardiología. Hospital Universitario San José. Popayán, Colombia.

${ }^{3}$ Especialista en Medicina Interna, Neumología, Oncología Torácica y Epidemiología. Docente del Departamento de Medicina Interna. Hospital Universitario San Vicente de Paúl Fundación. Medellín, Colombia.

${ }^{4}$ Especialista en Medicina Interna y Neumología. Docente del Departamento de Medicina Interna. Hospital Universitario San Vicente de Paúl Fundación. Medellín, Colombia.

${ }^{5}$ Especialista en Medicina Interna y Neumología. Docente del Departamento de Medicina Interna. Hospital Universitario San Vicente de Paúl Fundación. Medellín, Colombia.

${ }^{6}$ Especialista en Radiología. Hospital Universitario San Vicente de Paúl Fundación. Medellín, Colombia.

Autor de Correspondencia Juan David Cáceres González. Correo electrónico: caceres. juan@correounivalle.edu.co 


\section{Introducción}

La agenesia unilateral de la arteria pulmonar o interrupción de la arteria pulmonar $(1,2)$ es una rara anomalía congénita descrita inicialmente en 1868 por Fraentzel $(3,4,5)$. La prevalencia estimada es de 1 por cada 200.000 a 300.000 personas (6); compromete en igual frecuencia tanto a hombres como mujeres (7) y afecta principalmente la arteria pulmonar derecha siendo esta la de curso más benigno, al tener menos asociación con otras malformaciones $(8,9,10)$.

\section{Descripción detallada del caso}

Paciente femenina de 21 años con antecedente patológico de asma en la niñez que requería inhalación de salbutamol durante las crisis. Presentó un cuadro de neumonía seis meses antes de la consulta, que se manejó con esquema antibiótico por siete días. Sin antecedentes quirúrgicos ni toxicológicos de importancia. La paciente ingresa a la consulta ambulatoria con historia de dos meses de evolución de dolor en la espalda asociado a cuatro episodios de expectoración hemoptoica ocasional. Sin pérdida de peso, malestar general, sudoración, fiebre, alteración del apetito, ronquidos ni silbidos. Se encontraba alerta, consciente y orientada en tiempo, espacio y persona. Presentó frecuencia cardiaca de 72 latidos por minuto, frecuencia respiratoria de 18 por minuto, saturación de oxígeno de $95 \%$ al aire ambiente, presión arterial de 120/70 $\mathrm{mm} \mathrm{Hg}$ y afebril. Con ruidos cardiacos rítmicos sin soplos ni ruidos sobreagregados, murmullo vesicular conservado en ambos pulmones sin presencia de crépitos, sibilancias o roncus. El resto del examen físico era normal.

Para aclarar el diagnóstico se ordenó de manera ambulatoria: TAC contrastada de tórax, tomografía axial computarizada de alta resolución (TACAR) de tórax y espirometría pre y post broncodilatador. La TAC contrastada de tórax reportó presencia de tronco principal de la arteria pulmonar normal, ausencia de la arteria pulmonar izquierda (Figura 1) asociada con arco aórtico derecho y dilatación de las arterias bronquiales (Figura 2); prominencia de la arteria mamaria interna e intercostales ipsilaterales y circulación por colaterales transpleurales, una originándose desdela aorta a la altura del tronco celiaco. Vena cava normal, pulmón izquierdo hipoplásico con hiperinsuflación del pulmón derecho y desviación del cardiomediastino a la izquierda. Parénquima pulmonar izquierdo con algunos septos interlobulillares engrosados, sin bronquiectasia ni atenuación en mosaico (Figura 3).

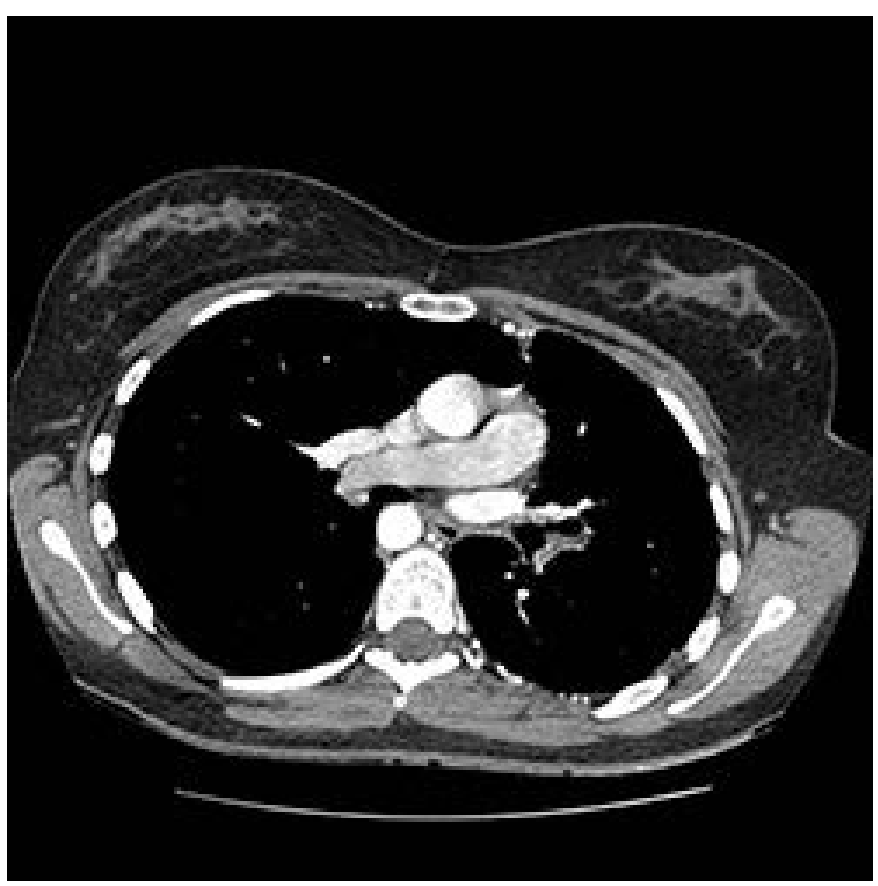

Figura 1. Tomografía axial computarizada contrastada de tórax, corte axial. Se observa la disrupción de la circulación de la arteria pulmonar por ausencia de la rama izquierda.

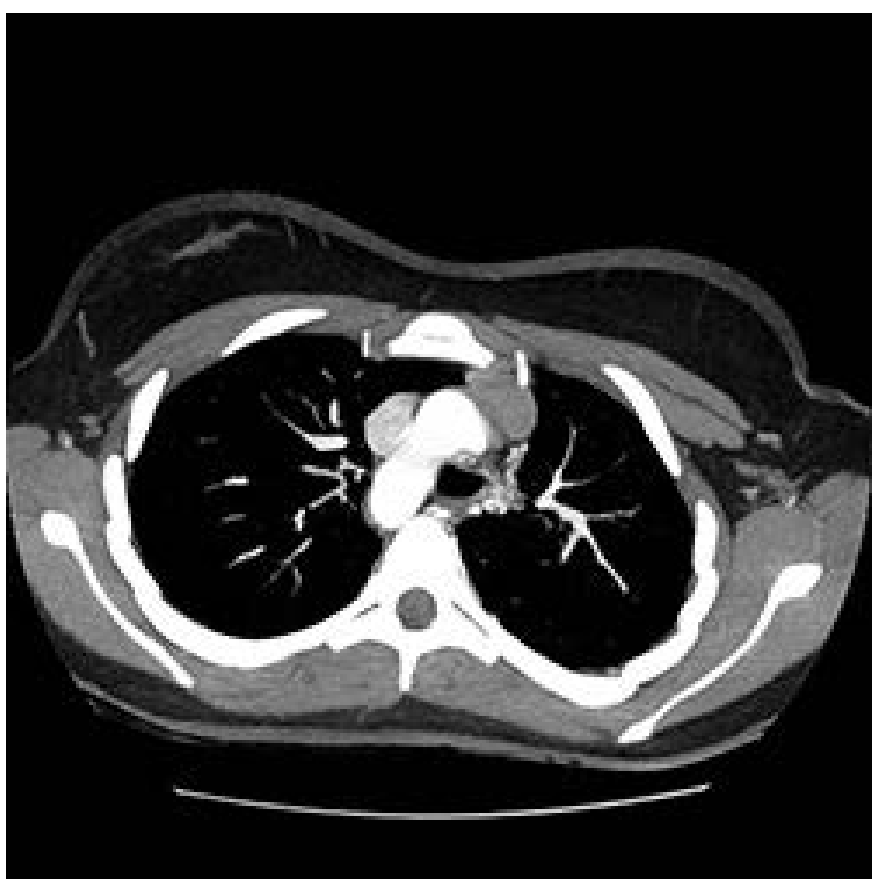

Figura 2. Tomografía axial computarizada contrastada de tórax, corte axial. Ausencia de arteria pulmonar izquierda con dilatación de las arterias bronquiales. 


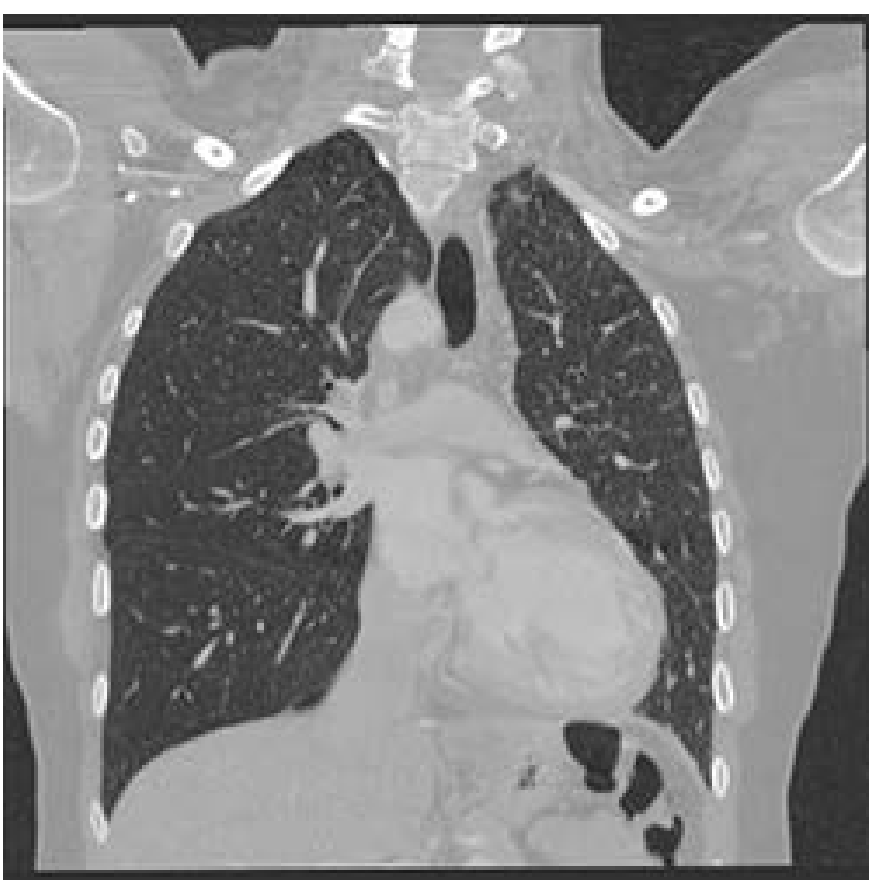

Figura 3. Tomografía axial computarizada contrastada de tórax ventana pulmonar, corte coronal. Pulmón izquierdo hipoplásico con hiperinsuflación del pulmón; parénquima pulmonar izquierdo con algunos septos interlobulillares engrosados, sin bronquiectasia ni atenuación en mosaico.

El mediastino anterior presentó imagen nodular con densidad de tejidos blandos de $30 \times 33 \mathrm{~mm}$ dado por hiperplasia tímica. Debido a la malformación vascular identificada en las imágenes, se decidió complementar el estudio con ecocardiograma transtorácico donde se encuentra función sistólica biventricular conservada, sin valvulopatías, sin hipertensión pulmonar, sin repercusión hemodinámica ni otras anomalías cardiacas asociadas. La paciente no se ha realizado gammagrafía de ventilación/perfusión. Se continuó el manejo para el asma con beclometasona (inhalador de $250 \mu \mathrm{g}$ ) 2 atomizaciones cada 12 horas y salbutamol (inhalador $100 \mu \mathrm{g}$ ) 2 atomizaciones a necesidad. La paciente durante el seguimiento ambulatorio presentó resolución de la tos hemoptoica con el manejo médico instaurado, sin requerimiento de intervención quirúrgica.

\section{Discusión}

La agenesia unilateral de la arteria pulmonar es un trastorno que se debe a una falla en la conexión entre el sexto arco aórtico que está involucionado y el tronco principal pulmonar durante el desarrollo embriológico $(4,7,9)$; por lo tanto, suele suplirse la ausencia de la irrigación hacia el lado afectado con una formación anómala de la vasculatura colateral (10). Esta malformación puede ir acompañada por ausencia completa o parcial del pulmón (11). Se asocia en su gran mayoría con otros trastornos cardiovasculares (8) como la tetralogía de Fallot, defecto septal ventricular, coartación aórtica, estenosis pulmonar, ductus arterioso persistente, estenosis aórtica subvalvular, arco aórtico del lado derecho, transposición de grandes vasos o síndrome de la cimitarra $(3,4,5,9,12)$. Cuando no se asocia con este tipo de trastornos, se considera como un hallazgo aislado $(8,9)$.

En el adulto suele pasarse inadvertido e incluso poco diagnosticado por los clínicos, debido a la amplia variedad de formas de manifestación $(13,14)$. La enfermedad puede ir desde un espectro asintomático o con síntomas inespecíficos $(6,8,10)$ hasta uno sintomático como la disnea de esfuerzo, tos persistente, hemoptisis en rara ocasiones, dolor torácico, infecciones respiratorias a repetición e hipertensión pulmonar $(4,5,6,8,12)$. Al examen físico puede haber como únicos hallazgos, que pueden pasar inadvertidos en la gran mayoría de los casos, disminución del murmullo vesicular y/o asimetría torácica $(4,8)$. Es gracias a ello que la sospecha debe estar presente ante un paciente que cursa con alguno de estos síntomas y así emplear exámenes complementarios que permitan aclarar el diagnóstico.

En este caso la paciente se encontraba con un examen físico sin alteraciones, pero con un antecedente importante de neumonía previa, acompañado de la hemoptisis, un hallazgo poco frecuente en este trastorno. Mediante la radiografía o tomografía de tórax puede observarse una opacidad pulmonar del lado afectado, disminución marcada de la vasculatura con elevación del hemidiafragma ipsilateral, desplazamiento del mediastino hacia el lado comprometido e hiperinsuflación compensatoria del lado contralateral $(10,11,12,15)$. Estos hallazgos imagenológicos pueden conllevar a un diagnóstico erróneo al simular tuberculosis, tumor pulmonar, tetralogía de Fallot, síndrome de Swyer-James-McLeod's, hipoplasia pulmonar, tromboembolismo pulmonar crónico o síndrome del pulmón hipogenético $(1,4,13)$.

La ecocardiografía es una herramienta necesaria para establecer el diagnóstico y descartar una afectación cardiaca asociada e hipertensión pulmonar $(7,8,15)$. Las pruebas de función pulmonar demuestran un patrón restrictivo leve con capacidad 
de difusión respiratoria normal $(6,10)$. La resonancia magnética nuclear y la TAC pueden ser de utilidad para visualizar el defecto vascular $(12,13,15)$. La angiografía, a pesar de ser la prueba diagnóstica estándar, (13) no se emplea de rutina, pero se indica su uso en aquellos pacientes que sean candidatos de revascularización o embolización, con el fin de caracterizar la lesión y evaluar la circulación colateral $(4,5,7,15)$.

El tratamiento en el paciente adulto dependerá de la presentación clínica y de la presencia de circulación colateral (13). Se puede ofrecer manejo sintomático en aquellos que cursen con hemoptisis debido a la alta tasa de autoresolución con el tiempo $(1,7)$. El manejo quirúrgico mediante lobectomía o neumonectomía con embolización selectiva se realiza en aquellos pacientes con hemoptisis masiva o infecciones pulmonares intratables (6-9, 13-14). Es preferible la revascularización en aquellos que tengan concomitantemente hipertensión pulmonar; de no ser posible, se podrían ofrecer medidas terapéuticas con vasodilatadores como inhibidores de la fosfodiesterasa, calcio antagonistas o antagonistas del receptor de endotelina $(7,14)$. Es necesario un manejo pertinente y temprano para evitar principales complicaciones como la falla cardiaca derecha, falla respiratoria, hemorragia pulmonar masiva, edema pulmonar grave o hipertensión pulmonar $(5,6,10)$.

\section{Conclusiones}

La agenesia unilateral de la arteria pulmonar sigue siendo una patología que por sus diversas formas de manifestación en el paciente y por su baja frecuencia encontrada en la población a nivel general, la convierte en un reto diagnóstico para el clínico. Se debe tener siempre presente la sospecha para abordar esta condición de manera oportuna, adecuada e integral, impactando significativamente en la calidad de vida del paciente que la presenta.

Declaración de fuentes de financiación y posibles conflictos de interés: Los autores no declaran conflictos de interés.

Agradecimientos: Al convenio entre la Universidad del Cauca y la Universidad de Antioquia por enriquecer y fortalecer la producción académica.

\section{Referencias}

1. Anand SH, Jasper A, Elizabeth Mani S, Joseph E, Mathai J. Proximal interruption of the pulmonary artery: a case series. J Clin Diagn Res. 2015 Dec;9(12):04-06. doi: 10.7860/ JCDR/2015/16198.6980

2. Irodi A, Prabhu SM, John RA, Leena RV. Congenital bronchopulmonary vascular malformations, "sequestration" and beyond. Indian J Radiol Imaging. 2015 Jan-Mar; 25(1):3543. doi: 10.4103/0971-3026.150138

3. Kumar Singhi EF, Raman Krishna K. Isolated absence of right pulmonary artery. Ann Pediatr Card. 2010;3(2).

4. Farghly E, Bousamra II M. Hemoptysis resulting from unilateral pulmonary artery agenesis. Ann Thorac Surg. 2002;74:255-7.

5. Takahashi T, Endo H, Ito T, Takei T, Yagi K. Isolated unilateral absence of the left pulmonary artery: a case report. Ann Vasc Dis. 2014;7(2):178182. doi: $10.3400 /$ avd.cr.14-00005

6. Ghobadi H, Matin S. Pulmonary artery agenesis with bronchial asthma. J Cardiothorac Med. 2014;2(2):172-175.

7. Mohan V, Mohan B, Tandon R, Kumbkarni S, Takkar Chhabra, S, Aslam N et al. Case report of isolated congenital absence of right pulmonary artery with collaterals from coronary circulation. Indian Heart J. 2014;66(2):220-222. https://doi. org/10.1016/j.ihj.2013.12.032

8. Volkan Emren SK, Yakar Tülüce S, Tülüce K. Isolated congenital unilateral agenesis of the left pulmonary artery with left lung hypoplasia in an asymptomatic adult patient. Acta Cardiol Sin. 2015;31:572-575. doi: 10.6515/acs20150511b

9. Flores M, Letter H, Derrick E, Koury I. Unilateral absence of the left pulmonary artery with an associated vascular anomaly in adulthood. Cureus 2016 Mar;8(3):e527. doi: 10.7759/cureus.527

10. Al Jabbari O, Abu Saleh WK, Ramchandani M, Scheinin S. Chronic recurrent unilateral pulmonary infection: result of congenital unilateral agenesis of pulmonary artery. 2016 Jan-Mar;12(1):48-50. doi: 10.14797/mdcj-12-148. 
11. Pinar Gunbey H, Gunbey E, Tanrivermis Sayit A, Bulut T. Unilateral right pulmonary agenesis in adulthood. J Clin Diagn Res. 2014 Jun;8(6):0102. doi: 10.7860/JCDR/2014/7968.4420

12. Karataş Z, Alp H, Altın H, Baysal T. Unilateral pulmonary artery agenesis: clinical and laboratory findings of four cases and diagnostic clues for pediatricians. Anadolu Kardiyol Derg. 2012 Nov;12:599-606. doi: 10.5152/akd.2012.194.

13. Hayek H, Palomino J, Thammasitboon, S. Right pulmonary artery agenesis presenting with uncontrolled asthma in an adult: a case report. J Med Case Rep. 2011;5:353.

14. Darwazah AK, Alhaddad IA. Pulmonary artery agenesis associated with coronary collaterals among adults. J Card Surg. 2016;11:109.

15. Griffin N, Mansfield L, Redmond KC, Dusmet M, Goldstraw P, Mittal TK et al. Imaging features of isolated unilateral pulmonary artery agenesis presenting in adulthood: a review of four cases. Clin Radiol. 2007;62:238-244. 